International Journal

of Sports Medicine

\title{
Reliability and validity of a self-paced cardiopulmonary exercise test in post-MI patients
}

\begin{tabular}{|c|c|}
\hline Journal: & International Journal of Sports Medicine \\
\hline Manuscript ID & IJSM-06-2016-5715-tt.R1 \\
\hline Manuscript Type: & Training \& Testing \\
\hline Key word: & cardiology, RPE, Aerobic Capacity, Pacing \\
\hline Abstract: & 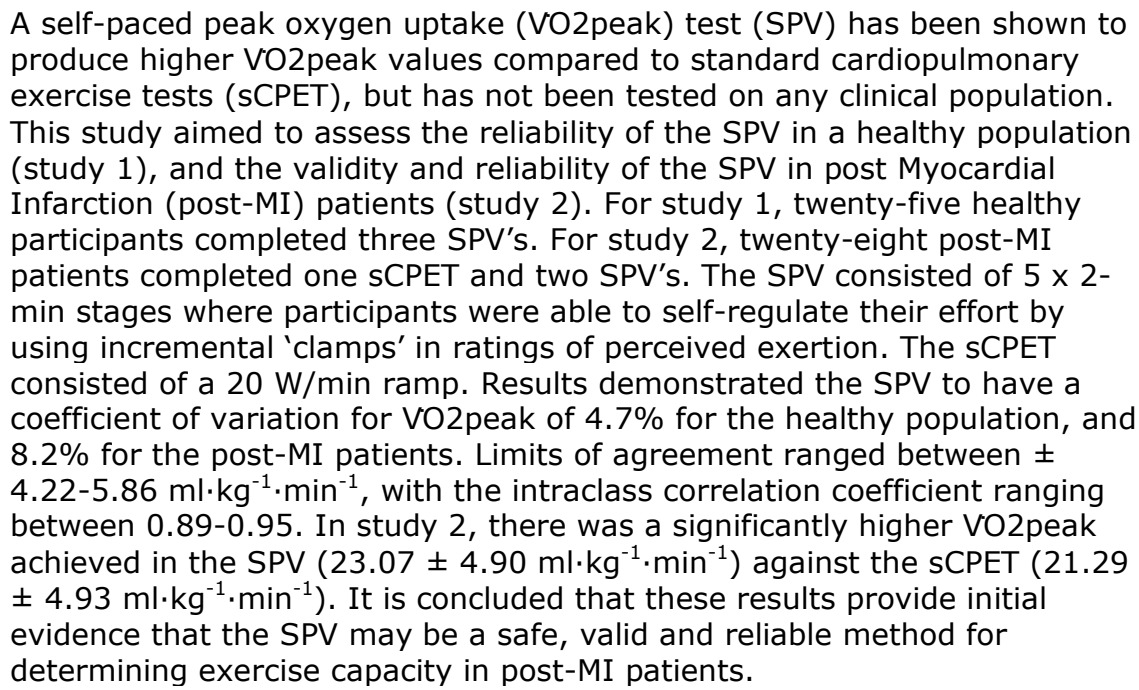 \\
\hline
\end{tabular}


1 Title: Reliability and validity of a self-paced cardiopulmonary exercise test in post-MI

2 patients

\section{Abstract}

4 A self-paced peak oxygen uptake $\left(\dot{\mathrm{V}}_{2 \text { peak }}\right)$ test $(\mathrm{SPV})$ has been shown to produce higher $5 \quad \dot{\mathrm{V}} \mathrm{O}_{2 \text { peak }}$ values compared to standard cardiopulmonary exercise tests (sCPET), but has not 6 been tested on any clinical population. This study aimed to assess the reliability of the SPV in

7 a healthy population (study 1), and the validity and reliability of the SPV in post Myocardial 8 Infarction (post-MI) patients (study 2). For study 1, twenty-five healthy participants 9 completed three SPV's. For study 2, twenty-eight post-MI patients completed one sCPET and 10 two SPV's. The SPV consisted of 5 x 2-min stages where participants were able to self11 regulate their effort by using incremental 'clamps' in ratings of perceived exertion. The 12 sCPET consisted of a $20 \mathrm{~W} / \mathrm{min}$ ramp. Results demonstrated the SPV to have a coefficient of 13 variation for $\mathrm{V}_{2 \text { peak }}$ of $4.7 \%$ for the healthy population, and $8.2 \%$ for the post-MI patients. 14 Limits of agreement ranged between $\pm 4.22-5.86 \mathrm{ml} \cdot \mathrm{kg}^{-1} \cdot \mathrm{min}^{-1}$, with the intraclass correlation 15 coefficient ranging between $0.89-0.95$. In study 2, there was a significantly higher $\dot{\mathrm{V}} \mathrm{O}_{2 \text { peak }}$ 16 achieved in the $\operatorname{SPV}\left(23.07 \pm 4.90 \mathrm{ml} \cdot \mathrm{kg}^{-1} \cdot \mathrm{min}^{-1}\right)$ against the $\mathrm{sCPET}\left(21.29 \pm 4.93 \mathrm{ml} \cdot \mathrm{kg}^{-}\right.$

$\left.17{ }^{1} \cdot \mathrm{min}^{-1}\right)$. We concludeIt is concluded that these results provide initial evidence that the SPV

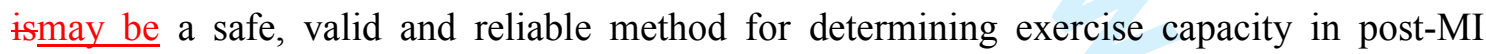
patients.

21 Key words: cardiology, RPE, aerobic capacity, pacing 


\section{Introduction}

27 Cardiopulmonary exercise testing (CPET) is an increasingly popular tool that allows 28 clinicians to objectively assess the integrated response to exercise $[29,48][27,41]$. Moreover, 29 CPET derived exercise tolerance and capacity have been strongly correlated with overall 30 health status and mortality, and can therefore provide valuable diagnostic and prognostic 31 information for various patient populations $[1,12,29,39][1,10,27,35]$. One of the key 32 measures obtained from CPET is peak oxygen uptake $\left(\mathrm{V}_{2 \text { peak }}\right)$, which is defined as the 33 highest amount of oxygen a person can utilise during dynamic exercise [7][5]. The 34 identification of $\dot{\mathrm{V}}_{2 \text { peak }}$ has become a fundamental procedure when assessing 35 cardiorespiratory fitness, monitoring exercise intensity [7][5] and when risk stratifying 36 individuals prior to major surgical procedures [8][6]. Exercise testing soon after a Myocardial 37 Infarction (MI) is beneficial as it can provide information on a patient's future risk of a 38 subsequent cardiac event [15][13] and can be used in individualising exercise rehabilitation 39 programmes [32][29].

41 Traditionally, CPET is completed on a stationary bike or a treadmill using a maximal 42 incremental exercise test (MIE), whereby the intensity (speed or power output (PO)) 43 increases by a set amount, for a given period of time, until volitional exhaustion is reached 44 [44][38]. For optimum values to be achieved it is suggested that participants reach volitional 45 exhaustion between 8-12 minutes [11][9]. Clinicians are therefore required to estimate the 46 most suitable starting intensity and work rate increments to ensure test validity. This 47 increases the risk of a test being unsuccessful due to participants either exceeding 12 minutes, 48 or worse, not lasting long enough for $\dot{\mathrm{V}}_{2 \text { peak }}$ to be accurately measured. There has also been 
49 a number of other limitations that have been brought to light regarding the general nature of the current CPET protocol [41], in particular the patient is unaware of the test duration and previous work suggests that knowledge of exercise duration can facilitate performance [33].

In addition the patient only has control over when they stop the test which adds a psychological aspect to the test i.e. low motivation [41]. Recently, a novel self-paced CPET protocol (SPV) was developed [36][32] to address the some of the aforementioned problems with the traditional CPET protocol. The SPV uses a closed-loop self-paced design, consisting of 5 x 2-min stages, where participants are able to regulate their work rate according to specific their-ratings of perceived exertion (RPE). Previous studies have concluded that the $\underline{\text { SPV is able to produce significantly higher } \dot{\mathrm{VO}}_{2 \text { peak }} \text { values when compared against traditional }}$ CPET protocols $[3,34,36]$, although not all studies have found this $[13,47]$. In recent years the $\underline{\text { SPV protocol has raised a lot of discussion points }[2,17,35,43] \text { with some researchers }}$ criticising the test $[13,17,43]$. Although, there is now a body of research which supports the validity of the SPV, with all studies demonstrating it to produce at least similar $\dot{\mathrm{VO}}_{2}$ peak values $[3,13,34,36,47]$. This type of test may be beneficial in clinical practice as it will reduce the risk of acquiring unusable data, this is because all patient will have the opportunity to complete the test at their own ability whilst meeting the recommended test time requirements.

A number of studies have assessed the use $[13,34,36,47][11,31,32,40]$, however the reliability of this protocol has yet to be determined. Moreover, no research has investigated the reliability or validity of the use of the SPV in a clinical population. There are a number of important benefits associated with completing $\underline{\text { CPET in post-MI patients }[15,32] \text {, therefore any test which may improve the validity and }}$ reliability of this process should be of interest. Therefore two separate studies werewe conducted two separate studies; 1 . To investigate the reliability of the SPV in an apparent 
74 "healthy" population, and; 2 . To investigate the reliability and validity of the SPV in early

75

76

77

78

79

80

81

82

83

84

85

86

87

post-MI patients. The hypotheses for the current study are that the SPV will be a reliable indicator of key CPET derived variables in the healthy and clinical populations. The SPV will also produce higher $\mathrm{VO}_{2 \text { peak }}$ values compared to a traditional CPET protocol in the post-MI patients.

\section{Materials \& Methods}

Twenty-five (12 females, 13 males) healthy participants (age $=26 \pm 6 \mathrm{yr}$, weight $=68 \pm 10$ $\mathrm{kg}$, height $=172 \pm 9 \mathrm{~cm}$ ) volunteered to participate in study 1 . Study 1 was conducted following institutional ethical approval of the researcher's own University. For study 2, $\underline{\text { thirty-seven patients undergoing phase III cardiac rehabilitation were asked to participate, out }}$ of those, thirty agreed to take part. Two patients withdrew from the study, therefore twentyeight post-MI patients ( 2 females, 26 males) undergoing cardiac rehabilitation volunteered to participate took park in study $2($ age $=58 \pm 8 \mathrm{yr}$, weight $=89.5 \pm 12 \mathrm{~kg}$, height $=178 \pm 8 \mathrm{~cm}$, days from MI event $57 \pm 35$ ). All participants recruited for study 2 already had their coronary angiography and any interventions needed following their MI, and were thought to require no further intervention or revascularisation. Study 2 was conducted following NHS ethical approval (Brighton and Sussex REC: 12/LO/1737). Both studies met the ethical standards outlined by Harris and Atkinson for the IJSM [21][18]. All participants gave their written informed consent.

For study 1 each participant visited the exercise-testing laboratory on three separate occasions. During each visit participants were required to complete an SPV test. For study 2 each patient was required to complete three exercise tests (a standard CPET protocol (sCPET) and two SPV tests) in order to determine the tests' validity and reliability. An 

overview of the experimental procedures for both studies are provided in figure 1. The order 100 in which participants completed the tests was in a randomised, counterbalanced crossover 101 design. For both studies, each test was separated by at least $24 \mathrm{~h}$ and all tests were completed

102

103

104 105

106

107

108

109

110

111

112 at the same time of the day $( \pm 2 \mathrm{~h})$. Participants were asked to refrain from drinking alcohol (24 $\mathrm{h}$ abstinence), eating ( $\mathrm{h}$ abstinence), smoking ( $2 \mathrm{~h}$ abstinence), and not to perform any exercise in the $24 \mathrm{~h}$ prior to each test. In both studies, participants were required to complete a 5-min warm-up at a self-selected intensity during which they were also familiarised with the process of freely adjusting their PO on the cycle ergometer.

\section{***INSERT FIGURE 1 HERE***}

The SPV was completed on an air-braked cycle ergometer (Wattbike Trainer, UK), which allowed participants to continually vary their PO throughout the test. The SPV was conducted in accordance with the procedures previously outlined by Mauger and Sculthorpe [36] [32] and consisted of $5 \times 2$-min stages (total test time of 10-min), where for each stage participants were able to continuously vary their PO, but with RPE (Borg's 6-20 scale) fixed to a level for each stage (RPE 11, 13, 15, 17 and 20), following an incremental format. Changes in PO were facilitated by the participants manually adjusting the cycle ergometer air brake and cadence at their own free will in order to produce a level of resistance that allowed them to match the target RPE for each stage of the SPV.

The sCPET from study 2 was completed on an electro-magnetically braked cycle ergometer (Lode Corival), so that PO for each stage could be fixed according to the test requirements. The test followed a standard incremental ramp design. As previously used with other clinical populations, the test commenced with no resistance and gradually increased by $20 \mathrm{~W}$ per 
minute, standardized across all patients $[10,16,20,26][8,14,17,24]$. The test was stopped when the patient felt like they could no longer continue or if they could no longer maintain more than 60 RPM, despite verbal encouragement.

During all exercise tests, expired gases were measured via the use of an online breath-bybreath analysis system (Cortex Metalyzer, Cortex, NL). Expired gases, heart rate (HR), PO and cadence were continuously recorded during the tests. A 12-lead ECG was used when exercising the post-MI patients in study 2. After the test, $\dot{\mathrm{V}} \mathrm{O}_{2 \text { peak }}$ was calculated as the highest 30 second average $\dot{\mathrm{V}} \mathrm{O}_{2}\left(\mathrm{~L} / \mathrm{min}^{-1}\right.$ and $\left.\mathrm{ml} \cdot \mathrm{kg}^{-1} \cdot \mathrm{min}^{-1}\right)$. We did not assess for a $\underline{\mathrm{A}} \dot{\mathrm{V}} \mathrm{O}_{2}$ plateau was not assessed which is why we the term $\dot{\mathrm{VO}}_{2 \text { peak }}$ is used, rather than $\dot{\mathrm{VO}}_{2 \text { max }}$. Peak cycling PO and minute ventilation (VंE) were also both calculated as the highest 30 second average value. The anaerobic threshold (AT) was determined using the V-slope method with confirmation via the ventilatory equivalents $\left(\dot{\mathrm{VE}} / \mathrm{VO}_{2}\right.$ and $\left.\mathrm{VE} / \dot{\mathrm{VCO}}_{2}\right)$ and the partial end-tidal $\left(\mathrm{P}_{\mathrm{ET}} \mathrm{O}_{2}\right.$ and $\left.\mathrm{P}_{\mathrm{ET}} \mathrm{CO}_{2}\right)$ methods [23][20]. All AT's were independently assessed by two experienced researchers.

All data was analysed using IBM SPSS Statistics version 21. Descriptive data is presented as mean \pm standard deviation $(\mathrm{SD})$. Statistical significance was set at $95 \%(\mathrm{p}<0.05)$. A sample size calculation was completed based upon the findings from the study by Mauger and

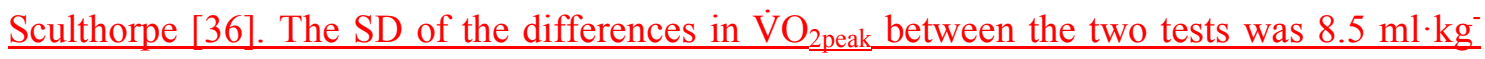
${ }^{1} \cdot \min ^{-1}[36]$ and if it is assumed that the minimal clinically worthwhile differences between the two tests is $5 \mathrm{ml} \cdot \mathrm{kg}^{-1} \cdot \mathrm{min}^{-1}$, this equals to an effect size of 0.58 . With this information it was therefore estimated that a minimim sample size of 25 was needed to achieve a statistical power $80 \%$ and an alpha level of 0.05 . Test-retest reliability was assessed via the use of $95 \%$ 
148 Limits of Agreement (LOA) using Bland-Altman plots [9][7], Confidence intervals (95\% CI) 149 of the coefficient of variation (CV), and intraclass correlation coefficients (ICC) were 150 calculated to assess the variability of the repeated tests (Hopkins, A New View of Statistics. 151 Internet Society for Sports Science: http://www.sportsci.org/resource/stats/index.html 152 (2015)). It has been suggested that a CV of $<5 \%$ [24][21], and an ICC close to 1 both 153 indicate good test-retest reliability [5][3], with classifications for ICC ranging from 154 'questionable' $(0.7$ to 0.8$)$ to 'high' $(>0.9)$ [5][3]. For study 1 differences in $\dot{\mathrm{V}} \mathrm{O}_{2 \text { peak, }}$, peak 155 PO, AT, peak HR and peak VंE were assessed using a one-way repeated measures ANOVA. 156 For study 2, physiological responses from the $1^{\text {st }}$ SPV test were compared to those obtained 157 from the sCPET, using a paired-samples t-test. Complete $2^{\text {nd }}$ SPV test data was not achieved 158 for three of the patients in study 2, and so data from only SPV1 has been used in these cases. 159 The reasons for these three missing tests were; one patient had an unrelated illness and was 160 unable to attend their final test within the required timeframe; the other two miscalculated 161 their work rate during the RPE 17 stage causing a premature end to the test. The data of these 162 two patients who did not meet the test requirements for SPV2 has been excluded from the 163 main analysis, but complete data is also presented within the results section.

165 Results

166 Study 1:

167 Table 1 represents a summary of the mean peak values for all the physiological variables 168 recorded during the three repeated SPVs. 169

***INSERT TABLE 1 HERE***

171 
172 The $\mathrm{CV}$ for $\dot{\mathrm{V}} \mathrm{O}_{2 \text { peak }}\left(\mathrm{ml} \cdot \mathrm{kg}^{-1} \cdot \mathrm{min}^{-1}\right)$ was $4.2 \%(95 \% \mathrm{CI}: 3.4-5.6 \%)$ for trials $2-1$ and $5.1 \%$ 173 (95\% CI: 4.2-6.8\%) for trials 3-2. The mean CV for all three tests was $4.7 \%$ (95\% CI: $3.8-$ 174 6.2\%). A high level of agreement was found between trials $2-1(\mathrm{ICC}=0.95)$ and trials 3-2 $175 \quad(\mathrm{ICC}=0.94)$. The LOA were $\pm 5.59 \mathrm{ml} \cdot \mathrm{kg}^{-1} \cdot \mathrm{min}^{-1}$ for trials 2-1 (Figure $\left.21 \mathrm{ta}\right)$ and \pm 5.86 $176 \mathrm{ml} \cdot \mathrm{kg}^{-1} \cdot \mathrm{min}^{-1}$ for trials $3-2$ (Figure $21 \mathrm{~b}$ ).

Participants demonstrated a mean CV of 5.5\% (95\% CI: 4.4-7.3\%) for AT, $7.9 \%(95 \% \mathrm{CI}$ : 6.3-10.6\%) for peak PO, $1.7 \%(95 \% \mathrm{CI}: 1.4-2.3 \%)$ for peak $\mathrm{HR}$, and $7.2 \%$ (95\% CI: $5.8-$ 180 9.6\%) for peak VंE. The ICC for these three variables ranged between 0.91-0.97.

\section{Study 2}

183 The CV for $\dot{\mathrm{V}}_{2 \text { peak }}$ between SPV1 and SPV2 was 8.2\% (95\% CI: 6.6-10.9\%). Therefore, if a 184 patient achieved a $\dot{\mathrm{VO}}{ }_{2 \text { peak }}$ of $23 \mathrm{ml} \cdot \mathrm{kg}^{-1} \cdot \mathrm{min}^{-1}$ a typical variation of $1.9 \mathrm{ml} \cdot \mathrm{kg}^{-1} \cdot \mathrm{min}^{-1}$ would 185 be expected. The ICC was 0.89 which represents a high level of agreement. The LOA was \pm $1864.22 \mathrm{ml} \cdot \mathrm{kg}^{-1} \cdot \mathrm{min}^{-1}$ for the measure of SPV1 and SPV2 (Figure 21 $1 \mathrm{c}$ ). If we include $\underline{\text { When the }}$ 187 SPV2 data for the two patients who were excluded from the main analysis are included, the CV becomes $8.4 \%$ (95\% CI: $6.8-11 \%$ ), the ICC is unchanged, and the LOA become \pm 4.52 $189 \mathrm{ml} \cdot \mathrm{kg}^{-1} \cdot \mathrm{min}^{-1}$.

190

191

***INSERT FIGURE 2 4 HERE***

192

193 The CV for AT between SPV1 and SPV2 was 8.4\% (95\% CI: 6.8-11.2\%). The ICC was 0.86 194 which suggests an 'acceptable' agreement [5][3]. The LOA was $\pm 3 \mathrm{ml} \cdot \mathrm{kg}^{-1} \cdot \mathrm{min}^{-1}$ for the 195 measure of SPV1 and SPV2 (Figure 21d). If we include the SPV2 data for the two patients 
196 who were excluded from the main analysis are included, the CV for AT becomes 8.6\% (95\% 197 CI: 7-11.4\%), the ICC is 0.84 , and the LOA remain unchanged. There was a CV of $15.1 \%$ 198 (95\% CI: $12.1-20.4 \%)$ for peak PO, $4.7 \%$ (95\% CI: $3.8-6.5 \%)$ for peak HR, and $11.5 \%(95 \%$ 199 CI: 9.2-15.4\%) for peak VंE. The ICC for these three variables ranged between 0.83-0.97, 200 demonstrating a high level of agreement $[5][3]$. 201

202 As shown in Table 2, patients achieved a significantly higher $\dot{\mathrm{VO}}_{2 \text { peak }}(\mathrm{p}<0.01)$ in the SPV 203 compared with the sCPET. Patients also achieved a significantly higher peak PO, peak HR 204 and peak $\dot{V} E$ in the SPV than in the $\operatorname{sCPET}(\mathrm{p}<0.01)$. There were no significant differences 205 in AT between the SPV and the sCPET $(p>0.05)$.

207

\section{Discussion}

This is the first study to assess the SPV on a clinical population. The results of the current 210 study demonstrated the SPV to be a reliable indicator of $\mathrm{V}_{2 \text { peak }}$ in a healthy population; 211 which is mirrored by the post-MI patient population. The $\mathrm{CV}$ for $\dot{\mathrm{V}} \mathrm{O}_{2 \text { peak }}\left(\mathrm{ml} \cdot \mathrm{kg}^{-1} \cdot \mathrm{min}^{-1}\right)$ in 212 the healthy population was $4.7 \%$ and $8.2 \%$ for the post-MI patients. Post-MI patients 213 achieved a higher $\dot{\mathrm{V}} \mathrm{O}_{2 \text { peak }}$ compared to a sCPET protocol, which is in agreement with 214 previous studies on healthy populations [34,36][31,32]. Previously published studies have 215 investigated the reproducibility of physiological variables using sCPET protocols. Froelicher 216 et al. [19][16] found that when using three popular maximal exercise treadmill protocols in a 217 healthy population the $\mathrm{CV}$ for $\dot{\mathrm{VO}}_{2 \text { peak }}$ ranged from $4.1-5.8 \%$. In addition, one study 218 completed a succession of CPET tests on cardiac failure patients and reported the average CV 219 for $\dot{\mathrm{V}} \mathrm{O}_{2 \text { peak }}$ to be 5.7\% [25][23]. Other studies have reported "good" test-retest reliability (CV 
220 for $\dot{\mathrm{V}} \mathrm{O}_{2 \text { peak }}=3.5-6.9 \%$ ) during cycling MIE tests in patients with various respiratory 221 conditions $[14,31,37][12,28,33]$. CV's from previous research [12,22,27,32] investigating the 222 use of traditional protocols are lower than those from the post-MI group of the current study. 223 However, it is difficult to make direct comparisons between studies as different patient 224 populations were used.

225

226 Our-The current study results demonstrated a CV for AT of 8.4\% (study 2), which is 227 considered as acceptable for test-retest reliability in clinical populations [40][36]. Kothmann 228 et al. [28][26] found a CV of 10\% for AT in Abdominal Aortic Aneurysm (AAA) patients 229 using a sCPET protocol. Identification of AT from CPET has become an increasingly 230 important tool in clinical exercise testing, primarily due to it giving an objective assessment 231 of cardiopulmonary function which does not require high levels of effort [42][37]. Previous 232 literature has demonstrated AT to be a useful predictor of mortality in patients with chronic 233 heart failure. This information can then be used to help prioritise patients for heart 234 transplantation [20][17]. The identification of AT prior to major surgery has also been shown 235 on a number of occasions to closely correlate with post-operative outcome $[42,49][37,42]$. It

236 is reassuring to see that in the current study there were no differences in AT when comparing 237 it between the SPV and the SCPET $(p>0.05)$, this combined with the reliability results 238 demonstrate that AT can be reliably determined via the SPV, which is of great importance in 239 clinical exercise testing.

240

241 As previously mentioned, two post-MI patients were excluded from the main reliability 242 analysis as they did not successfully complete a second SPV due to misjudging the required 243 work rate during stage RPE 17. However, when looking at their individual test data, both 
244 patients exercised long enough to demonstrate a valid AT and a $\dot{\mathrm{V}} \mathrm{O}_{2 \text { peak. }}$. When including 245 their exercise data into the reliability analysis the $\mathrm{CV}$ for $\dot{\mathrm{V}} \mathrm{O}_{2 \text { peak }}$ increases from 8.2 to $8.4 \%$ and $\mathrm{CV}$ for AT increases from 8.4 to $8.6 \%$. From a clinical perspective it is encouraging to see that even though these two patients did not complete the full 10-min, important CPET 248 data could still be obtained from the test.

In agreement with data from a healthy population [36][32], post-MI patients achieved a 251 significantly higher $\dot{\mathrm{VO}}_{2 \text { peak }}(+8 \%)$ during the SPV compared with the sCPET $(\mathrm{P}<0.01)$.

252 Peak HR and VंE were also significantly higher in the SPV than in the SCPET $(p<0.01)$, 253 which is in support of previous work [18,22,34][15,19,31]. It is interesting to see that 254 previous studies which failed to find any differences in $\dot{\mathrm{VO}}_{2 \text { peak }}$ between the SPV and a 255 SCPET protocol also found no differences in HR and VE $[13,47][11,40]$, potentially leading to the observed differences in $\dot{\mathrm{VO}}_{2 \text { peak }}$. A recently published study [3][2] found significantly higher maximal HR and cardiac output during the SPV compared to a SCPET protocol [3][2].

Astorino et al. $[3][2]$ concluded that the greater cardiac output in the SPV suggests a greater oxygen delivery to the exercising muscles, permitting a higher $\mathrm{V}_{2 \text { peak }}$ to be achieved. These findings suggest that the SPV allows individuals to work to a higher physiological work rate when compared to the sCPET. This may be a result of the nature of self-paced exercise providing a more "comfortable" experience for patients. Previous research has in fact suggested that self-paced exercise is less physiologically challenging when compared against enforced paced exercise [30]. Being able to make slight adjustments in effort may minimise fatigue and any peripheral discomfort associated with cycling, particularly in the early stages 266 of the test, which may ultimately lead to a greater work rate being able to be achieved in the 267 final stage [3][2]. In traditional CPET no adjustments in effort can be made and the only way 268 to stop any exercise related discomfort would be to stop. In addition, it may be that 
269 knowledge of the test end-point in the SPV also contributes to the higher work rates achieved. Indeed, previous literature has demonstrated that knowledge of exercise duration can improve exercise performance [33]. With all of this in mind, the current findings suggest that in a clinical population, where cardiac function might be limited, the self-paced nature may in fact provide the patient the opportunity to work harder, producing a greater cardiac output and therefore reaching a higher $\dot{\mathrm{V}} \mathrm{O}_{2 \text { peak. }}$. However, further research is required to support theseis speculations.

The mean sCPET time-to-exhaustion was 8 minutes 55 seconds (range $=5 \min -12 \min 54$ sec) compared to the fixed 10 minutes of the SPV. Even though the sCPET mean test time falls within the recommended criteria of 8-12 minutes [11][9], only 15 (of 28) participants successfully completed the test within this recommended time. Therefore, the lower $\dot{\mathrm{V}} \mathrm{O}_{2 \text { peak }}$ 281 in the sCPET could be attributable to only 54\% achieving the recommended test time. A 282 potential limitation of the current study was the decisionthat we decided to standardize sCPET work rate increments $(20 \mathrm{~W} / \mathrm{min})$ for all patients $[10,16,26][8,14,24]$ instead of doing so oan an individual basis $[38,39][34,35]$. Individualising work rate increments may have resulted in more patients completing the CPET within the recommended time frame, although the subjectivity of such a choice would not have guaranteed a successful test in all patients. This issue clearly highlights one of the key challenges practitioners face on a day-to-day basis 288 when using CPET with clinical populations. Indeed, if patients are unable to exercise for a 289 sufficient time the utility of test results is severely limited, resulting in a significant waste of 290 finance and time for both patients and health service provider. In particular, an incorrect estimation of the work rate increments may lead to a test which is too short, or too long. A test which is short in duration $(<8 \mathrm{~min})$ is suggested to underestimate $\dot{\mathrm{V}}_{2 \text { peak }}$ due to 
294 [4]. In addition to this, a short test may only acquire limited information making it difficult to 295 confidently assess fitness. Conversely, if a test is long in duration ( $>12 \mathrm{~min}$ ) patients may end up stopping due to such factors as boredom or increased local muscle fatigue [4], rather than a result of their actual cardiopulmonary limit. The SPV eliminates the need for practitioners to estimate the most appropriate starting intensity and work rate increments as it is based on set levels of perceived exertion. Moreover, the closed loop nature of the SPV 300 ensures that each test lasts 10 minutes. The nature of the SPV gives patients the opportunity 301 to complete the test at their own ability whilst exercising for the recommended time to achieve optimal physiological values. This therefore may increase the likelihood of obtaining useable and representative data from patients. Thus, a protocol like the SPV may be a more 304 reliable way of acquiring time efficient and useable data than sCPET methods.

The significantly higher PO achieved in the SPV suggests that regardless of the self-paced nature, participants were willing to tolerate significantly higher work rates in the final stage (RPE 20), compared to that demanded by the sCPET. Knowledge of the test end-point in the SPV vs the open-ended SCPET could contribute to the higher tolerance in work rate. Indeed, previous literature has demonstrated that knowledge of exereise duration can improve exercise performance [30]. It could also be suggested that the SPV provides patients with a more "comfortable" experience, as they are able to self adjust their work rate, potentially making the higher perceived work rates more tolerable, especially when the end is proximate.

314 There were no adverse events reported for the current study, providing support for the current 315 evidence base that maximal exercise testing is a safe procedure to perform on cardiac patients \begin{tabular}{l|l}
316 & {$[6,27,46][4,25,39]$.} \\
317 &
\end{tabular} 
\begin{tabular}{l|l}
318 & A limitation of the current study is that different cycle ergometers were used in study 2. \\
319 & Previous research has suggested that different ergometers might result in differences in the \\
320 & metabolic and cardiovascular response [45]. However, different ergometers were a \\
321 & requirement of the different protocols, as the SPV required patients to freely adjust their PO, \\
322 & and the sCPET required accurate fixing of PO. Indeed, a similar differences in $\mathrm{VO}_{2 \text { peak }}$ to that \\
323 & seen in the current study have been found by previous studies who used the same cycle \\
324 & ergometer in both the SPV and sCPET [3,36]. \\
325 &
\end{tabular}

326 Conclusion

327 The results of the current study demonstrate that the SPV is a reliable method for determining

$328 \dot{\mathrm{V}} \mathrm{O}_{2 \text { peak }}$ in a healthy population, with acceptable reproducibility being seen in the clinical

329 population. The SPV allowed post-MI participants to achieve a significantly higher $\dot{\mathrm{V}} \mathrm{O}_{2 \text { peak }}$

330 than the sCPET. This study provides initial evidence suggests that the SPV may be is a safe,

331 valid and reliable measure of $\dot{\mathrm{V}} \mathrm{O}_{2 \text { peak }}$ in both clinical and healthy populations, and should be

332 considered as an accepted means of testing for exercise capacity. However, further robust 333 multicentre data is required to establish the safety of the SPV in such populations. Moreover,

334 the defined test duration and self-administered work rates associated with the SPV addresses

335 common issues that clinicians regularly have to overcome, and go some way to ensuring all

336 patients exercise for the recommended duration in order to obtain a valid and reliable CPET.

337 Future research should seek to assess the SPV in other clinical populations and the utility of

338 the SPV versus SCPET to inform clinical decision making on patient treatment.

339

340 Acknowledgements

Georg Thieme Verlag KG. P. O. Box 3011 20, D-70451 Stuttgărt, Germany. http://www.thieme.de/fz/sportsmed/index.html 
341 We firstly thank Dr Marcus Fletcher for his clinical support for the tests conducting in study

342 2. We also thank the cardiac rehabilitation nurses at the William Harvey Hospital for their 343 help with recruiting suitable patients and test supervisions. We finally thank Dr. Katharine 344 Richardson for assisting in interpretation of the Anaerobic Threshold data.

Funding: This work was supported by the East Kent Hospitals Charity [1076555/303 to $347 \mathrm{JGH}]$.

Disclosures: None.

350

351

References

352

1. Albouaini K, Egred M, Alahmar A, Wright DJ. Cardiopulmonary exercise testing and its 353 application. Heart 2007; 93: 1285-1292.

2. Astorino TA. Discussion: The efficacy of the self-paced VO2max test to measure maximal 355 oxygen uptake in treadmill running. Appl Physiol Nutr Metab 2014; 39: 592-593.

3. Astorino TA, McMillan DW, Edmunds RM, Sanchez E. Increased cardiac output elicits 357 higher VO2max in response to self-paced exercise. Appl Physiol Nutr Metab 2015; 40: 223358229.

4. Astorino TA, Rietschel JC, Tam PA, Taylor K, Johnson SM, Freedman TP, Sakarya CE. 360 
361 5. Atkinson G, Nevill AM. Statistical methods for assessing measurement effort (reliability) in 362 variables relevant to sports medicine. Sports Med 1998; 26: 217-238.

363

364

365 366

6. Balady GJ, Arena R, Sietsema K, Myers J, Coke L, Fletcher GF, Forman D, Franklin B, Guazzi M, Gulati M, Keteyian SJ, Lavie CJ, Macko R, Mancini D, Milani RV, on behalf of the American Heart Association Exercise, Cardiac Rehabilitation, and Prevention Committee of the Council on Clinical Cardiology, Council on Epidemiology and Prevention, Council on Peripheral Vascular Disease, Interdisciplinary Council on Quality of Care and Outcomes Research. Clinician's Guide to Cardiopulmonary Exercise Testing in Adults: A Scientific Statement From the American Heart Association. Circulation 2010; 122: 191-225.

7. Basset D, Howley ET. Limiting factors for maximum oxygen uptake and determinants of endurance performance. Med Sci Sports Exerc 2000; 32: 70-84.

8. Beckles MA, Spiro SG, Colice GL, Rudd RM. The physiologic evaluation of patients with lung cancer being considered for resectional surgery. Chest 2003; 123: 105-114.

9. Bland MJ, Altman DG. Statistical methods for assessing agreement between two methods of clinical measurement. Lancet 1986; 327: 307-310.

10. Brutsche MH, Spiliopoulos A, Bolliger CT, Licker M, Frey JG, Tschopp JM. Exercise capacity and extent of resection as predictors of surgical risk in lung cancer. Eur Respir $\mathbf{J}$ 2000; 15: 828-832.

11. Buchfuhrer MJ, Hansen JE, Robinson TE, Sue DY, Wasserman K, Whipp DJ. Optimizing the exercise protocol for cardiopulmonary assessment. J Appl Physiol 1983; 55: 1558-1564. 
381 12. Buys R, Cornelissen V, Van De Bruaene A, Stevens A, Coeckelberghs E, Onkelinx S, 382 Thomaes T, Delecluse C, Budts W, Venhees L. Measures of exercise capacity in adults with 383 congenital heart disease. Int J Cardiol 2011; 153: 30.

18. Faulkner J, Mauger AR, Woolley B, Lambrick DM. The efficacy of a self-paced VO2max 400

13. Chidnok W, DiMenna FJ, Bailey SJ, Burnley M, Wilkerson DP, Vanhatalo A, Jones AM. VO2max is not altered by self-pacing during incremental exercise. Eur J Appl Physiol 2013; 113: 529-539.

14. Cox NJM, Hendrink JCM, Binkhorst RA, Folgering HTM, van Herwaarden CLA. Reproducibility of incremental maximal cycle ergometer tests in patients with mild to moderate obstructive lung disease. Lung 1989; 167: 129-133.

15. Davidson DM, DeBusk RF. Prognostic value of a single exercise test 3 weeks after uncomplicated myocardial infarction. Circulation 1980; 61: 236-242.

16. Eindhoven JA, van den Bosch AE, Oemrawsingh RM, Baggen VJ, Kardys I, Cuypers JA, Witsenburg M, van Schaik RH, Roos-Hesselink JW, Boersma E. Release of growthdifferentiation factor 15 and associations with cardiac function in adult patients with congenital heart disease. Int J Cardiol 2016; 202: 246-251.

17. Eston RG, Crockett A, Jones AM. Discussion of: The efficacy of the self-paced V` O2max test to measure maximal oxygen uptake in treadmill running. Appl Physiol Nutr Metab 2014; 39: $581-582$. test during motorized treadmill exercise. Int J Sports Physiol Perform 2015; 10: 99-105. 
401 19. Froelicher VF, Brammell H, Davis G, Noguera I, Stewart A, Lancaster MC. A 402 comparison of the reproducibility and physiologic response to three maximal treadmill 403 exercise protocols. Chest 1974; 65: 512-517.

404 20. Gitt AK, Wasserman K, Kilkowski C, Kleemann T, Kilkowski A, Bangert M, Schneider S, 405 Schwarz A, Senges J. Exercise anaerobic threshold and ventilator efficiency identify heart 406 failure patients for high risk of early death. Circulation 2002; 106: 3079-3084.

407 21. Harriss DJ, Atkinson G. Ethical standards in sport and exercise science research: 2016 408 update. Int J Sports Med 2015; 36: 1121-1124.

409 22. Hogg JS, Hopker JG, Mauger AR. The self-paced VO2max test to assess maximal oxygen 410 uptake in highly trained runners. Int J Sports Physiol Perform 2014; 10: 172-177.

411 23. Hopker JG, Jobson SA, Pandit JJ. Controversies in the physiological basis of the 412 'anaerobic threshold' and their implications for clinical cardiopulmonary exercise testing. 413 Anaesthesia 2011; 66: 111-123.

414 24. Hopkins WG. Measures of reliability in sports medicine and science. Sports Med 2000; $415 \quad 30: 1-15$.

416 25. Janicki JS, Gupta S, Ferris ST, McElroy PA. Long-term reproducibility of respiratory gas 417 exchange measurements during exercise in patients with stable cardiac failure. Chest 1990; $418 \quad 97: 12-17$.

419 26. Kahaly GJ, Wagner S, Nieswandt J, Mohr-Kahaly S, Ryan TJ. Stress Echocardiography 420 in Hyperthyroidism. J Clin Endocrinol Metab 1999; 84: 2308-2313. 
421 27. Kanthan A, Tan TC, Zecchin RP, Denniss AR. Early exercise stress testing is safe after 422 primary percutaneious coronary intervention. Eur Heart J Acute Cardiovasc Care 2012; 1: $423 \quad 153-157$.

424 28. Kothmann E, Danjoux G, Owen SJ, Parry A, Turley AJ, Batterham AM. Reliability of the 425 anaerobic threshold in cardiopulmonary exercise testing of patients with abdominal aortic 426 aneurysms. Anaesthesia 2009; 64: 9-13.

427 29. Kristensen SD, Knuuti J, Saraste A, Anker S, Botker HE, Hert SD, Ford I, Gonzalez428 Juanatey JR, Gorenek B, Heyndrickx GR, Hoeft A, Huber K, Lung B, Kjeldsen KP, Longrois 429 D, Luscher TF, Pierard L, Pocock S, Price S, Roffi M, Sirnes PA, Sousa-Uva M, Voudris V, 430 Funck-Bentano C. 2014 ESC/ESA Guidelines on non-cardiac surgery: cardiovascular 431 assessment and management. Eur Heart J 2014; 35: 2383-2431.

432 30. Lander PJ, Butterly RJ, Edwards AM. Self-paced exercise is less physically challenging 433 than enforced constant pace exercise of the same intensity: influence of complex central 434 metabolic control. Br J Sports Med 2009; 43: 789-795.

435 31. Marciniuk DD, Watts RE, Gallagher CG. Reproducibility of incremental maximal cycle 436 ergometer testing in patients with restrictive lung disease. Thorax 1993; 48: 894-898.

437 32. Markiewicz W, Houston N, DeBusk RF. Exercise testing soon after myocardial infarction. 438 Circulation 1977; 56: 26-31. 33. Mauger AR, Jones AM, Williams GA. Influence of feedback and prior experience on 440 pacing during a 4-km cycle time trial. Med Sci Sports Exerc 2009; 41: 451-458. 
441 34. Mauger AR, Metcalfe AJ, Taylor L, Castle PC. The efficacy of the self-paced VO2max 442 test to measure maximal oxygen uptake in treadmill running. Appl Physiol Nutr Metab 2013; $443 \quad 38: 1211-1216$.

444 35. Mauger AR, Metcalfe AJ, Taylor L, Castle PC. Reply to "Discussion: Efficacy of the self445 paced VO2max test to measure maximal oxygen uptake in treadmill running". Appl Physiol $446 \quad$ Nutr Metab 2014; 39: 583-585.

447 36. Mauger AR, Sculthorpe N. A new VO2max protocol allowing self-pacing in maximal 448 incremental exercise. Br J Sports Med 2012; 46: 59-63.

37. McKone EF, Barry SC, FitzGerald MX, Gallagher CG. Reproducibility of maximal exercise ergometer testing in patients with cystic fibrosis. Chest 1999; 116: 363-368.

38. Myers J, Buchanan N, Walsh D, Kraemer M, McAuley P, Hamilton-Wessler M, Froelicher VF. Comparison of the ramp versus standard exercise protocols. JACC 1991; 17: $1334-1342$.

39. Myers J, Prakash M, Froelicher V, Partington S, Atwood E. Exercise capacity and mortality among men referred for exercise testing. N Engl J Med 2002; 346: 793-801.

40. Myers J, Goldsmith RL, Keteyian SJ, Brawner CA, Brazil DA, Aldred H, Ehrman JK, Burkhoff D. The Ventilatory Anaerobic Threshold in Heart Failure: A Multicenter Evaluation of Reliability. J Card Fail 2010; 16: 76-83.

41. Noakes TD. Testing for maximum oxygen consumption has produced a brainless model of human exercise performance. Br J Sports Med 2008; 42: 551-555. 
461 42. Older P, Hall A, Hader R. Cardiopulmonary exercise testing as a screening test for 462 perioperative management of major surgery in the elderly. Chest 1999; 116: 355-362.

463

464

465

466

467

468

469

470

471

472

473

474

475

476

477

478

479

480

481

43. Poole DC. Discussion: "The efficacy of the self-paced $\mathrm{V}^{\cdot} \mathrm{O} 2 \mathrm{max}$ test to measure maximal oxygen uptake in treadmill running”. Appl Physiol Nutr Metab 2014; 39: 586-588.

44. Poole DC, Wilkerson DP, Jones AM. Validity of criteria for establishing maximal $\mathrm{O}_{2}$ uptake during ramp exercise tests. Eur J Appl Physiol 2008; 102: 403-410.

45. Reiser M, Meyer T, Kindermann W, Daugs R. Transferability of workload measurements between three difference types of ergometer. Eur J Appl Physiol 2000; 82: 245-249.

46. Roffi M, Wenaweser P, Windecker S, Mehta H, Eberli FR, Seiler C, Fleisch M, Garachemani A, Pedrazzini GB, Hess OM, Meier B. Early exercise after coronary stenting is safe. J Am Coll Cardiol 2003; 42: 1569-1573.

47. Straub AM, Midgley AW, Zarvorsky GS, Hillman AR. Ramp-incremented and RPEclamped test protocols elicit similar $V \mathrm{O}_{2 \max }$ values in trained cyclists. Eur J Appl Physiol 2014; 114: 1581-1590.

48. West $M$, Jack $S$, Grocott $M P W$. Perioperative cardiopulmonary exercise testing in the elderly. Best Pract Res Clin Anaesthesiol 2011; 25: 427-437.

49. Wilson RJT, Davies $S$, Yates D, Redman J, Stone M. Impaired functional capacity is associated with all-cause mortality after major elective intra-abdominal surgery. Br J Sports Med 2010; 105: 297-303.

\section{Figure legend:}


482

483 Figure 1. GraphicalSchematic overview forof the experimental proceduresprotocols foref

$484 \quad$ study 1 and 2.

485

486

Figure 2. Bland-Altman plots of a) differences in $\dot{\mathrm{VO}}_{2 \text { peak }}$ between trials 1 and 2 from study 1;

487 b) trials 2 and 3 from study 1; c) differences in $\dot{\mathrm{V}}_{2 \text { peak }}$ between SPV1 and SPV2 from study

488 2; d) differences in AT between SPV1 and SPV2 from study 2. The solid horizontal line

489 represents mean difference, whilst the dashed lines represent the 95\% LOA.

490

Georg Thieme Verlag KG. P. O. Box 3011 20, D-70451 Stuttgart, Germany. http://www.thieme.de/fz/sportsmed/index.html 
Table 1: Peak values for physiological variables recorded during repeated SPV tests in the healthy population.

\begin{tabular}{|c|c|c|c|}
\hline & SPV1 & SPV2 & SPV3 \\
\hline$\dot{\mathrm{V}} \mathbf{O}_{2 \text { peak }}\left(\mathrm{L} / \mathrm{min}^{-1}\right)$ & $3.30 \pm 0.86$ & $3.23 \pm 0.90$ & $3.25 \pm 0.92$ \\
\hline$\dot{\mathbf{V}} \mathbf{O}_{2 \text { peak }}\left(\mathrm{ml} \cdot \mathrm{kg}^{-1} \cdot \mathrm{min}^{-1}\right)$ & $48.56 \pm 8.93$ & $47.87 \pm 9.28$ & $47.85 \pm 9.40$ \\
\hline $\operatorname{AT}\left(\mathrm{ml} \cdot \mathrm{kg}^{-1} \cdot \mathrm{min}^{-1}\right)$ & $27.00 \pm 6.83$ & $26.67 \pm 7.26$ & $26.95 \pm 7.34$ \\
\hline HR (bpm) & $184 \pm 10$ & $183 \pm 11$ & $182 \pm 11$ \\
\hline$\dot{\mathbf{V}} \mathbf{E}\left(\mathrm{L} / \mathrm{min}^{-1}\right)$ & $137.8 \pm 38.9$ & $133.3 \pm 41.0$ & $128.4 \pm 39.1^{*}$ \\
\hline Peak PO (Watts) & $312 \pm 93$ & $299 \pm 109$ & $304 \pm 101$ \\
\hline
\end{tabular}

*significantly different to SPV1 $(<0.05)$, data are mean \pm SD. 
Table 2: Physiological variables recorded over the sCPET, SPV1 and SPV2 in post-MI patients.

\begin{tabular}{|c|c|c|c|}
\hline & sCPET $(n=28)$ & SPV1 $(n=28)$ & SPV2 $(n=25)$ \\
\hline$\dot{\mathbf{V}} \mathbf{O}_{2 \text { peak }}\left(\mathrm{L} / \mathrm{min}^{-1}\right)$ & $1.90 \pm 0.50$ & $2.05 \pm 0.48^{*}$ & $2.00 \pm 0.43$ \\
\hline$\dot{\mathrm{V}} \mathbf{O}_{2 \text { peak }}\left(\mathrm{ml} \cdot \mathrm{kg}^{-1} \cdot \mathrm{min}^{-1}\right)$ & $21.29 \pm 4.93$ & $23.07 \pm 4.90^{*}$ & $22.68 \pm 4.79$ \\
\hline $\mathrm{AT}\left(\mathrm{ml} \cdot \mathrm{kg}^{-1} \cdot \mathrm{min}^{-1}\right)$ & $12.63 \pm 2.41$ & $13.06 \pm 2.39$ & $13.21 \pm 2.76$ \\
\hline HR (bpm) & $129 \pm 18$ & $138 \pm 14^{*}$ & $136 \pm 19$ \\
\hline$\dot{V} E\left(L / \min ^{-1}\right)$ & $82.0 \pm 27.1$ & $94.5 \pm 25.9^{*}$ & $91.1 \pm 26.2$ \\
\hline Peak PO (Watts) & $171 \pm 43$ & $209 \pm 78^{*}$ & $200 \pm 64$ \\
\hline TTE (seconds) & $535 \pm 130$ & $600 \pm 0^{*}$ & $600 \pm 0$ \\
\hline
\end{tabular}

*significantly different from the sCPET $(<0.05)$, data are mean \pm SD. 


\section{Study 1 (healthy):}

\begin{tabular}{|c|c|c|c|c|c|}
\hline \multirow[t]{2}{*}{ SPV } & RPE 11 & RPE 13 & RPE 15 & RPE 17 & RPE 20 \\
\hline & $2 \min$ & $2 \min$ & $2 \min$ & $2 \min$ & $2 \min$ \\
\hline
\end{tabular}

\section{Study 2 (post- MI patients):}

$$
\begin{aligned}
& \text { sCPET (visit } 1 \text { or 2) } \operatorname{Ramp~} 20 \mathrm{~W} / \mathrm{min}^{-1}
\end{aligned}
$$

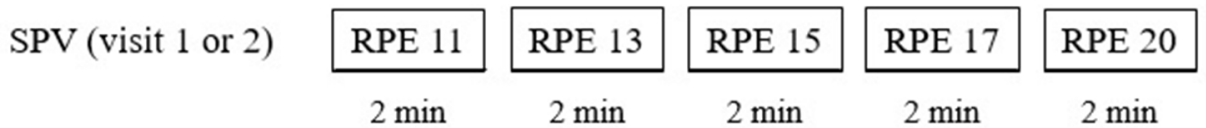

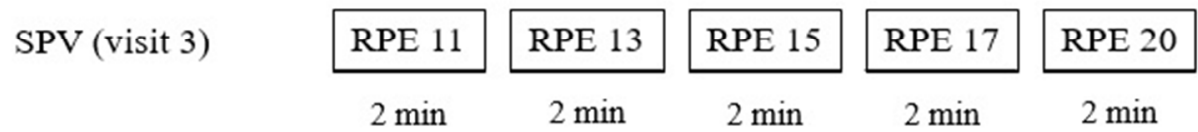

Figure 1 . Schematic of the experimental protocols for study 1 and 2 .

$$
175 \times 121 \mathrm{~mm}(96 \times 96 \mathrm{DPI})
$$



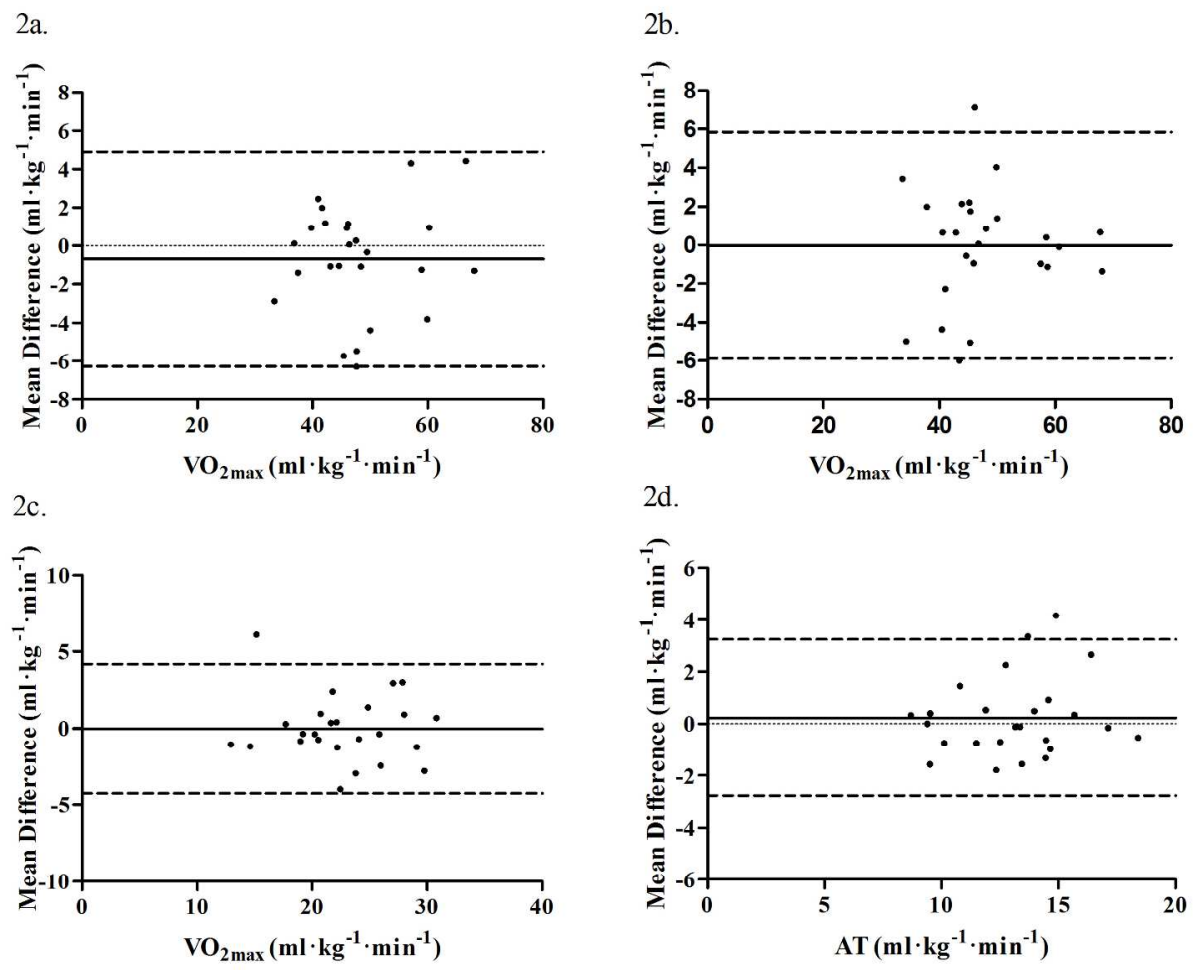

Figure 2. Bland-Altman plots of a) differences in VO2peak between trials 1 and 2 from study 1 ; b) trials 2 and 3 from study $1 ;$ c) differences in VO2peak between SPV1 and SPV2 from study 2; d) differences in AT between SPV1 and SPV2 from study 2. The solid horizontal line represents mean difference, whilst the dashed lines represent the 95\% LOA. $F_{T}$

$224 \times 178 m m(300 \times 300$ DPI $)$ 